\title{
A Low-Complexity Synchronization Design for MB-OFDM Ultra-Wideband Systems
}

\author{
Zhenzhen Ye, Chunjie Duan, P. Orlik, Jinyun Zhang
}

TR2008-032 May 2008

\begin{abstract}
In this paper, we investigate the low-complexity synchronization design for multi-band orthogonalfrequency-division-multiplexing (MB-OFDM) ultra-wideband (UWB) systems. We propose a unified synchronizer design based on auto-correlation-function. The key component in the proposed synchronizer is a parallel signal detector structure in which multiple auto-correlation units are instantiated and their outputs are shared by other functional units in the synchronizer, including time-frequency pattern detection, symbol timing carrier frequency offset estimation and correction and frame synchronization. We show that, via analysis and simulations, such a design achieves not only a low computation cost which makes it attractive in implementation, but also equal or better performance compared to the cross-correlation based designs.
\end{abstract}

IEEE International Conference on Communications (ICC)

This work may not be copied or reproduced in whole or in part for any commercial purpose. Permission to copy in whole or in part without payment of fee is granted for nonprofit educational and research purposes provided that all such whole or partial copies include the following: a notice that such copying is by permission of Mitsubishi Electric Research Laboratories, Inc.; an acknowledgment of the authors and individual contributions to the work; and all applicable portions of the copyright notice. Copying, reproduction, or republishing for any other purpose shall require a license with payment of fee to Mitsubishi Electric Research Laboratories, Inc. All rights reserved. 



\section{A Low-Complexity Synchronization Design for MB-OFDM Ultra-wideband Systems}

\author{
Zhenzhen Ye \\ Dept. of Electrical, Computer \& Systems Engineering \\ Rensselare Polytechnic Institute \\ Troy, NY USA \\ Email: yez2@rpi.edu
}

\author{
Chunjie Duan, Philip Orlik, Jinyun Zhang \\ Mitsubishi Electric Research Labs \\ 201 Broadway \\ Cambridge, MA USA \\ Email: \{duan, porlik, jzhang\}@merl.com
}

\begin{abstract}
In this paper, we investigate the low-complexity synchronization design for multi-band orthogonal-frequencydivision-multiplexing (MB-OFDM) ultra-wideband (UWB) systems. We propose a unified synchronizer design based on auto-correlation-function. The key component in the proposed synchronizer is a parallel signal detector structure in which multiple auto-correlation units are instantiated and their outputs are shared by other functional units in the synchronizer, including time-frequency pattern detection, symbol timing, carrier frequency offset estimation and correction and frame synchronization. We show that, via analysis and simulations, such a design achieves not only a low computation cost which makes it attractive in implementation, but also equal or better performance compared to the cross-correlation based designs.
\end{abstract}

\section{INTRODUCTION}

The past few years have seen Multi-band OrthogonalFrequency-Division-Multiplexing (MB-OFDM) Ultrawideband (UWB) becoming the leading player in highdata-rate, short-range wireless personal area network (WPAN) thanks to its high spectrum efficiency, robustness and outstanding performance under diverse environments. WiMedia $^{1}$ has published a series of standards and its PHY/MAC specification has been adopted by ECMA as an international standard [1].

Since many targeted applications involve mobile/portable devices, low-power, low-complexity solutions are essential for the prevalence of this technology. The receiver synchronization circuit (SYNC) has been identified as one of the most powerconsuming circuits in the baseband. This is due to it's higher active duty cycle than other baseband components. Also the performance of the SYNC has direct implication on the overall system performance as errors introduced by the SYNC (misses in acquisition, estimation error in timing and carrier frequency offset etc.) degrade the overall system performance rapidly.

Unlike most other baseband blocks in the transceiver, the SYNC carries out signal processing in the time domain and therefore is referred to as a pre-FFT ${ }^{2}$ processing unit [2], [3]. The SYNC is a multi-task unit and contains several functional blocks that perform: detection of the arrival of the synchronization symbols; identification of the time-frequency code (TFC); determination of the start of the FFT window,

\footnotetext{
${ }^{1}$ WiMedia is an open industry association that promotes MB-OFDM UWB.

${ }^{2}$ FFT stands for fast Fourier transform
}

estimation of the carrier frequency offset (CFO) and data frame synchronization.

Research on the SYNC design has been active for over a decade, either for general OFDM transceiver structures [14], [2], [6], [7], [4], [11], [9] or for specific communication systems using OFDM modulation [3], [13], [15], [12], [10]. Most existing work focus on optimizing one or two functional blocks that comprise the SYNC circuitry and assume that the other blocks work perfectly. To the best of our knowledge, there is no existing work comprehensively studying a unified structure for all the blocks in the SYNC. More importantly, most works have not fully explored the complexity issue in MB-OFDM UWB synchronizer design. For example, a matched filter is still widely used in synchronization symbol detection [10], [15] and symbol timing [15], [12], even though it is known to have a high computation cost and requires a large amount of hardware resources.

This work presents a complete SYNC design specific for low-power, low-complexity implementation of the MB-OFDM UWB system. Our proposed design uses a parallel autocorrelator structure which is shared by all the functional blocks including signal detection, TFC identification, symbol timing, CFO estimation and etc. Both theoretical analysis and simulation show that such an architecture enables a low-cost implementation without compromising performance.

The remainder of the paper is organized as follows: Section II gives the system model of the MB-OFDM UWB transceiver. It also outlines the difference of auto-correlation function and cross-correlation function in computation cost and implementation complexity; In Section III, we present the overall architecture of the proposed SYNC design, followed by the details of the design and the operation of individual functional units. The simulation results and performance analysis are given in Section $\mathrm{IV}^{3}$. Section V draws the conclusion.

\section{SySTEM DESCRIPTION}

\section{A. An MB-OFDM Signal Model in Synchronization}

In [1], a transmitted symbol is defined as an OFDM symbol with $N=128$ sub-carriers followed by $N_{g}=32$ samples of zero-padding. An additional $\left(N_{g}^{\prime}-N_{g}\right) T=5$ sample periods

\footnotetext{
${ }^{3}$ Due to the space limitation, we skip the derivations for many performances analysis results listed below and refer the interested reader to [16].
} 
are inserted for RF band switching where the sampling interval $T \sim 2 n s$. The resulting OFDM symbols are of total length $T_{s}=\left(N+N_{g}^{\prime}\right) T$, which includes $N_{s}=N+N_{g}^{\prime}=165$ samples. Each OFDM symbol contains $Q<N$ data symbols $a_{l, k}$, where $l$ denotes the OFDM symbol time index and $k \in$ $[-Q / 2,-1] \cup[1, Q / 2]$ denotes the subcarrier frequency index ${ }^{4}$, the transmitted baseband signal is given by ${ }^{5}$

$$
s(t)=\frac{1}{\sqrt{N T}} \sum_{l} \sum_{k} a_{l, k} e^{j 2 \pi(k / N)(t-l T)} u(t-l T)
$$

where $u(t)=1,0 \leq t<N T$ and zero otherwise.

The equivalent baseband frequency selective fading channel including the actual UWB channel impulse response (CIR) at the corresponding frequency band as well as the effect of analog path of the transceiver can be modeled as

$$
h(\tau)=\sum_{i} h_{i} \delta\left(\tau-\tau_{i}\right),
$$

where the complex path gains $\left\{h_{i}\right\}$ and the path delays $\left\{\tau_{i}\right\}$ are assumed to be time-invariant within one data frame [1]. The maximum path delay is $\tau_{\max }$. Taking into account the CFO between the transmitter and the receiver, the discrete received baseband signal can be expressed as

$$
r[m]=e^{j 2 \pi m \Delta f T} \sum_{i} h_{i} s\left(m T-\tau_{i}\right)+\nu[m]
$$

where $\nu[m]$ is the complex zero-mean white Gaussian noise with the variance $\sigma_{\nu}^{2}, \Delta f$ is the frequency offset.

For channel estimation and data demodulation, the receiver performs the overlap-and-add (OLA) operation on the received symbols and then demodulates the data via FFT. When a symbol timing bias $d$ is taken into account ${ }^{6}$, the $l$ th OFDM symbol for FFT operation is given by $\mathbf{r}_{l}=\left[r_{l, 0}+\right.$ $\left.r_{l, N}, r_{l, 1}+r_{l, N+1}, \ldots, r_{l, N_{g}-1}+r_{l, N+N_{g}-1}, r_{l, N_{g}}, \ldots, r_{l, N-1}\right]$ where $r_{l, m}=r\left(\left(m+d+l N_{s}\right) T\right), m=0,1, \ldots, N-1$.

\section{B. A Low-Complexity Consideration}

Before looking at any specific design, we would like to first identify two basic operations widely used in OFDM synchronization design for detecting repeated, pre-defined signals:

1) cross-correlation function $(\mathrm{CCF})$ : $C C(d)=\sum_{m} r^{*}[m+d] s[m]$, in which the received signal $\{r[m]\}$ is correlated with a known synchronization symbol $\{s[m]\}$;

2) auto-correlation function (ACF): $A C(d)=\sum_{m} r^{*}[m+d] r[m+M+d]$, in which the correlation is carried out between the segments of the received signals with different delays, i.e., $\{r[m]\}$ and $\{r[m+M]\}$.

\footnotetext{
${ }^{4}$ The DC component in OFDM symbols is set to 0 .

${ }^{5}$ Note that (1) only models the OFDM symbols on one frequency band. Since the transmissions/receptions on three bands are independent and symmetric, this model is valid as long as our concern is the reception of an individual OFDM symbol.

${ }^{6}$ The imperfection of the baseband sampling clock also has effect on the system behavior, however, it is insignificant during the preamble period [16] From here on, we assume a perfect sampling clock in synchronization.
}

Even though two functions looks similar, there is a significant difference in computation complexity between them. The $\mathrm{ACF}$ can be rewritten into an iterative structure as follows

$$
\begin{aligned}
A C(d) & =\sum_{m=1}^{K} r^{*}[m+d] r[m+M+d] \\
& =A C(d-1)+U[K+d]-U[d],
\end{aligned}
$$

where $U[d] \triangleq r^{*}[d] r[M+d]$. Therefore, the ACF only requires 1 complex multiplication (for calculating $U[K+d]$ ) and 2 complex additions for every new sample. It can be readily implemented in hardware using one complex multiplier, two adders and some delay elements (e.g., memory). On the other hand, no such efficient implementation is available for CCF. In the case of MB-OFDM UWB synchronization symbol $\{s[m]\}$ with the length of 128 samples, CCF requires 256 real multiplications (or 64 complex multiplications) and 127 complex additions per sample, much more expensive than an ACF-based design.

For complexity and power efficiency, an ACF-based SYNC is definitely appealing. The concern, however, is that a pure ACF-based design may suffer performance degradation. In the following sections, we show that such a design does not compromise the system performance. On the contrary, the robustness to frequency offsets make it more attractive than the CCF-based design.

\section{AN ACF-BASED SYNCHRONIZER DESIGN}

\section{A. The Overall SYNC Structure}

We propose an ACF-based SYNC structure as shown in Figure 1. Our design is not only motivated by the complexity issue discussed above, but also based on two unique characteristics of WiMedia's MB-OFDM UWB signal design: the time-frequency hopping pattern of MB-OFDM UWB signal (Table I) and the structure of the preamble of a data packet defined in [1]. The preamble of a data packet consists of 24 repeated synchronization symbols. The symbols are 128-point time-domain real pseudo-random (PR) sequences, unique to given TFCs. That is the synchronization symbols are repetitions of a particular code and we note that they do not undergo any OFDM modulation. The 128-point codes are then zero padded with 37 zero samples so that a single symbol is the same length as an OFDM symbol (165 samples). The repeated synchronization symbols make the ACF a natural choice in design and the TFC patterns in Table I motivate us to adopt a parallel ACF structure. The proposed parallel ACF structure can not only achieve a rapid acquisition and TFC identification (see Section III-B), but also is important in designing an iterative CFO estimator as discussed in Section III-D.

In the parallel ACF block, there are four ACF units that performs auto-correlation between the input signal and signals that are delayed by $1,3,5$ and 6 symbols length. The outputs of the ACF blocks can be expressed as

$$
A C(d ; p, W) \triangleq \sum_{m=0}^{N+W-1} r^{*}[m+d] r\left[m+p N_{s}+d\right]
$$




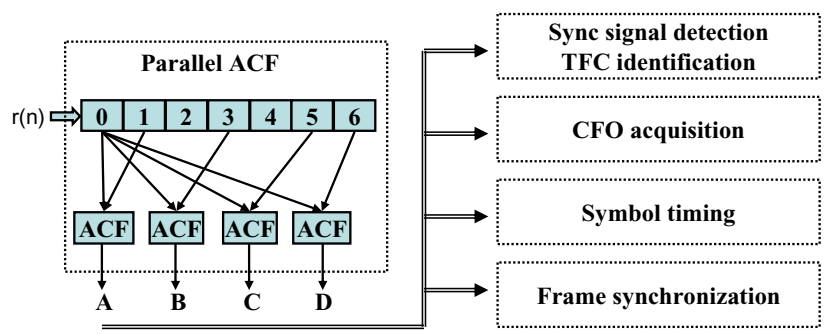

Fig. 1. Top-level structure of a parallel ACF based synchronization block. Numbers in delay blocks indicate synchronization symbol delay, i.e., a delay of 165 samples.

where $p$ has the values of $1,3,5,6$ respectively and $N+$ $W(W \geq 0)$ controls the length of the received signal segments that are processed with auto-correlation operations. The hardware to implement the parallel ACF block involves 4 complex multipliers, 8 adders and delay elements. Note that this is only a small fraction of a single 128-point CCF implementation.

During the synchronization, each ACF unit outputs one value for every new input sample. The outputs are fed into all functional blocks. We describe the operation details of individual blocks in the following sections.

TABLE I

Time-FreQuency CODES [1]

\begin{tabular}{|c|c|c|c|c|c|c|}
\hline TFC Number & \multicolumn{6}{|c|}{ Hopping pattern } \\
\hline \hline 1 & 1 & 2 & 3 & 1 & 2 & 3 \\
\hline 2 & 1 & 3 & 2 & 1 & 3 & 2 \\
\hline 3 & 1 & 1 & 2 & 2 & 3 & 3 \\
\hline 4 & 1 & 1 & 3 & 3 & 2 & 2 \\
\hline 5 & 1 & 1 & 1 & 1 & 1 & 1 \\
\hline 6 & 2 & 2 & 2 & 2 & 2 & 2 \\
\hline 7 & 3 & 3 & 3 & 3 & 3 & 3 \\
\hline
\end{tabular}

\section{B. Signal Detection and TFC Identification}

Before the synchronization is acquired and TFC is identified, a receiver needs to scan through all sub-bands, i.e., it stays on one band and "listens" to possible incoming UWB signals for a period of time. If no packet is detected, the receiver switches to a different band and continues listening. For any incoming packet, because of the frequency hopping, only the symbols in the sub-band that the receiver is listening can be "heard". As an example, for TFC-1 listed in Table I, the receiver listening on sub-band 1 receives only one of every 3 preamble symbols. With the proposed parallel ACF structure, as soon as its output pattern (after comparing to a given threshold) matches the ones given in Table II, the detection of signal is declared. Note that the minimum Hamming distances of different output patterns in Table II is 2 and thus one error in the output pattern can be detected. The output $\mathbf{D}$ not only improves the robustness of the detection but also is needed in refining CFO estimation.

As shown in Table II, the detector output pattern also indicates the TFC group of the detected signal. If the received signal belongs to TFC $1-4$, additional steps are needed to determine the TFC. In this case, to determine which TFC 1,
TABLE II

Output Patterns of the Parallel ACF Signal Detector, After COMPARING TO A GIVEN THRESHOLD.

\begin{tabular}{|c|c|c|c|c|}
\hline TFC group & A & B & C & D \\
\hline \hline TFC 1,2 & 0 & 1 & 0 & 1 \\
\hline TFC 3,4 & 1 & 0 & 0 & 1 \\
& 0 & 0 & 1 & 1 \\
\hline TFC 5-7 & 1 & 1 & 1 & 1 \\
\hline
\end{tabular}

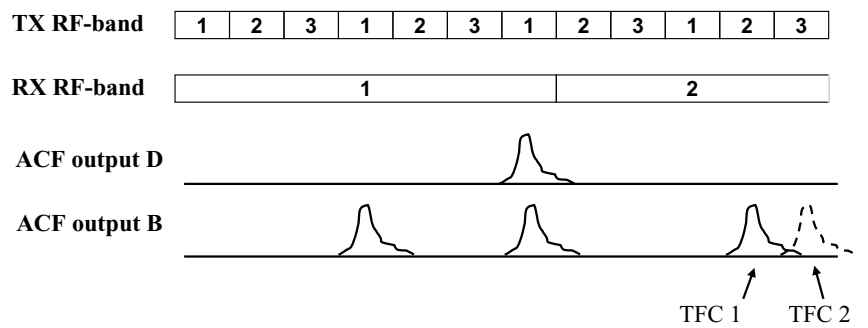

Fig. 2. An illustration on the ACF-based TFC identification via band-

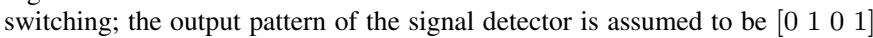
( $B=1, D=1$ ), i.e., the possible TFC is either 1 or 2 ; the ACF peak position uniquely determines the TFC of the received signal.

2,3 , or 4 the transmitter is using, the receiver can do one of the following:

1) perform CCF between the received signal segment and one of the known synchronization symbols in the group of the TFCs; the TFC is determined by comparing the CCF value with a pre-defined threshold; or

2) switch to a different sub-band and continue to perform ACF operations on the incoming baseband signal and determine the TFC by identifying the location of the ACF output peaks.

The first scheme is more computationally intensive and requires additional hardware. In addition, even though the different PR sequences of synchronization symbols have a good auto-correlation property, their cross-correlation property is fairly poor. This increases the difficulty in setting the threshold in the first scheme. On the other hand, the second scheme avoids the difficulty in setting an absolute threshold, is computationally cheaper and, more importantly, the existing hardware (i.e., ACF units) are reused. The procedure is illustrated in Figure 2. Assuming the output pattern $\left[\begin{array}{llll}0 & 1 & 0 & 1\end{array}\right]$ is detected on sub-band 1, the receiver switches to sub-band 2 and continues to do ACF operations on two signal segments with the separation of $3 N_{s} T$. The peak position of the ACF outputs implies the TFC of the received signal.

\section{OFDM Symbol Timing}

After the preamble signal is detected and its TFC is identified, the SYNC needs to search for the start of an OFDM symbol, i.e., symbol timing. Inaccurate timing not only introduces inter-carrier-interference (ICI) and (possibly) inter-symbol-interference (ISI), but also affects the quality of channel estimation and the signal energy collected in the FFT window. It has significant implication on system bit-error-rate (BER). The optimal symbol timing point should maximize the 
output SNR after the FFT. For illustration, consider the MBOFDM UWB signal of TFC 1 or 2 where ISI due to timing error is negligible ${ }^{7}$, we have [16]

$$
d_{\text {opt }}=\arg \max _{d}\left\{\frac{\sigma_{s}^{2}}{\sigma_{I C I}^{2}+\left(1+\frac{N_{g}}{N}\right) \sigma_{\nu}^{2}}\right\}
$$

where $d$ is the timing bias (in the unit of sampling period, $T)$, w. r. t. the start of a received OFDM symbol, $\sigma_{s}^{2}$ is the signal power and $\sigma_{I C I}^{2}$ is the ICI power after FFT operation. Specifically,

$$
\begin{aligned}
\sigma_{s}^{2} & =\int_{0}^{\eta} E\left[|h(\tau)|^{2}\right]\left(1-\frac{\eta-\tau}{N T}\right)^{2} d \tau+\int_{\eta}^{\eta+T_{g}} E\left[|h(\tau)|^{2}\right] d \tau \\
& +\int_{\eta+T_{g}}^{\tau_{\max }} E\left[|h(\tau)|^{2}\right]\left(1-\frac{\tau-\eta-T_{g}}{N T}\right)^{2} d \tau, \\
\sigma_{I C I}^{2} & =\int_{0}^{\eta} E\left[|h(\tau)|^{2}\right]\left(1-\frac{\eta-\tau}{N T}\right)\left(\frac{\eta-\tau}{N T}\right) d \tau \\
& +\int_{\eta+T_{g}}^{\tau_{\max }} E\left[|h(\tau)|^{2}\right]\left(1-\frac{\tau-\eta-T_{g}}{N T}\right)\left(\frac{\tau-\eta-T_{g}}{N T}\right) d \tau,
\end{aligned}
$$

with $\eta \triangleq d T$, i.e., the timing bias in continuous time domain. A close-formed solution to (5) is difficult to obtain. Alternatively, we look at a slightly different optimization criterion which maximizes the difference between the signal power and the sum of ICI and noise power, i.e.,

$$
\hat{d}_{o p t}=\arg \max _{d}\left\{\sigma_{s}^{2}-\sigma_{I C I}^{2}-\left(1+\frac{N_{g}}{N}\right) \sigma_{\nu}^{2}\right\} .
$$

It can be shown that $\hat{d}_{o p t}$ approximately satisfies [16]

$$
\int_{0}^{\hat{d}_{o p t} T} E\left[|h(\tau)|^{2}\right] d \tau=\int_{\hat{d}_{o p t} T+T_{g}}^{\tau_{\max }} E\left[|h(\tau)|^{2}\right] d \tau .
$$

This can be interpreted as the optimal timing point $\hat{d}_{\text {opt }}$ approximately equalizes channel energy in $\left[0, \hat{d}_{\text {opt }} T\right]$ and $\left[\hat{d}_{o p t} T+T_{g}, \tau_{\max }\right]$. We note that if $\tau_{\max } \leq T_{g}$, the channel energy out of $\left[d T, d T+T_{g}\right]$ is zero when $d$ is optimal and thus (7) is trivially satisfied.

In practice, the symbol timing schemes for OFDM systems generally can be classified as CCF-based [13], [12], ACFbased [3], [6], [7] and hybrid [14], [15], [10]. Both the hybrid and CCF-based metric are computationally intensive and the CCF-based one is also sensitive to frequency offset, even though the hybrid one is expected to have the best performance and resilience to narrow-band interference [14], [15], [10]. Numerous ACF-based timing schemes have been proposed in the literature [9], [3], [6], [7]. and all can be implemented with low-complexity. The major difference among the schemes is in normalizing/biasing the auto-correlation values to meet the different criteria [9]. Here we use the maximum correlation (MC) metric [9] in the proposed synchronizer, i.e.,

$$
\hat{d}_{1}=\arg \max _{d}\left\{\left|A C\left(d ; p, N_{g}\right)\right|\right\} .
$$

In [16], we have shown that the metric (8) satisfies (7) and thus can achieve a near-optimal performance.

\footnotetext{
${ }^{7}$ The analysis on TFC 3-7 where ISI is non-negligible can be manipulated in exactly the same way in [16] with a slightly more tedious derivation.
}

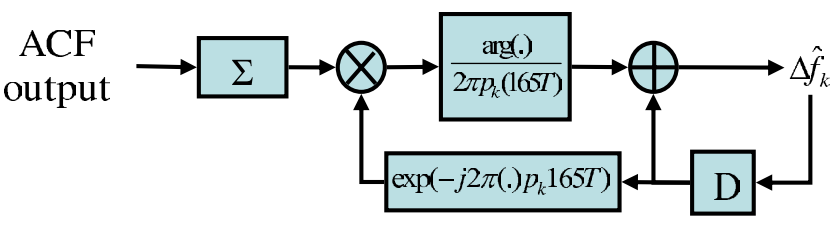

Fig. 3. The structure of iterative CFO estimator, where $N_{s}=165$.

TABLE III

ITERATIVE CFO ESTIMATION

\begin{tabular}{l}
\hline \hline (1) Known: $A C\left(d ; p_{1}, N_{g}\right), d \in \Gamma_{1}, p_{1} \geq 1$ \\
(1a) $S_{1, \Gamma_{1}}=\sum_{d \in \Gamma_{1}} A C\left(d ; p_{1}, N_{g}\right) ;$ \\
(1b) $\Delta \hat{f}_{1}=\frac{1}{2 \pi p_{1}\left(N+N_{g}\right) T} \arg \left\{S_{1, \Gamma_{1}}\right\} ;$ \\
(2) For $k \geq 2$, known $A C\left(d ; p_{k}, N_{g}\right), d \in \Gamma_{k}, p_{k}>p_{k-1}$ \\
(2a) $S_{k, \Gamma_{k}}=\sum_{d \in \Gamma_{k}} A C\left(d ; p_{k}, N_{g}\right) e^{-j 2 \pi \Delta \hat{f}_{k-1} p_{k} N_{s} T ;}$ \\
(2b) $\delta \hat{f}_{k}=\frac{1}{2 \pi p_{k}\left(N+N_{g}\right) T} \arg \left\{S_{k, \Gamma_{k}}\right\} ;$ \\
(2c) $\Delta \hat{f}_{k}=\Delta \hat{f}_{k-1}+\alpha \delta \hat{f}_{k} ;$ \\
(3) Go back to (2) for further refinement on the estimation. \\
\hline \hline
\end{tabular}

\section{Carrier Frequency Offset (CFO) Estimation}

Another task of the SYNC is to estimate the carrier frequency offset (CFO) so the CFO can be compensated before the receiver starts channel estimation and data demodulation. In the proposed SYNC, the CFO estimation can be carried out in parallel with symbol timing. The CFO w.r.t. the absolute central frequency is specified to be within $\pm 20 \mathrm{ppm}$ [1] and therefore, the maximum relative CFO $\Delta f$ between two nodes is $\pm 40 \mathrm{ppm}$ (This translates into $\sim 160 \mathrm{kHz}$ for a carrier center frequency of $4 \mathrm{GHz}$ ). CFO estimation is typically done in two steps: pre-FFT and post-FFT. The pre-FFT operation gives initial CFO estimation, which is critical as it affects the following channel estimation and data demodulation, is typically implemented as part of synchronization. Once the system is synchronized, the residual CFO can be removed via pilot-tone tracking in the frequency domain, the so called postFFT CFO tracking.

A commonly used scheme for CFO estimation in the timedomain is based on estimating the phases of the ACF values close to the maximum value of $\left|A C\left(d ; p, N_{g}\right)\right|$ [3], [12], [6]. The value of $p$ indicates the delay interval between two correlated symbols. On one hand, a small $p$, allows us to estimate a large range of $\mathrm{CFO}$ without phase ambiguity since the phase of an $\mathrm{AC}$ value $A C\left(d ; p, N_{g}\right)$ is given by $p 2 \pi N_{s} \Delta f T$. On the other hand, a large $p$ yields a high frequency resolution. To satisfy both range and accuracy requirements, we need to use ACF outputs with multiple $p$ values.

Based on this observation, we propose a general CFO estimation algorithm which has an iterative structure and takes advantage of different values of $p$. The details of the algorithm are given in Table III, where $\Gamma_{k}$ is the set of timing points close to the peak of $\left|A C\left(d ; p_{k}, N_{g}\right)\right|$ and $\alpha \in(0,1]$ is the stepsize for updating the estimation in each iteration. The basic structure of such a CFO estimator is illustrated in Fig. $3(\alpha=1)$. 


\section{E. Frame Synchronization}

The preamble uses a frame synchronization cover sequence to modulate the polarities of the 24 synchronization symbols [1]. The goal of frame synchronization is to synchronize the receiver to the cover sequence. Generally this process starts when the TFC identification, symbol timing and CFO estimation/correction are completed and the receiver frequency hopping is already enabled.

A frame synchronization design based on the ACF outputs of two adjacent symbols in the same band is much simpler than one with CCF since there is no accumulative phase rotation in the ACF outputs. A negative peak in the ACF output indicates a polarity change in the cover sequence. For improved robustness, multiple ACFs (with different delays) can be used. For example, the cover sequence for TFC-1 has

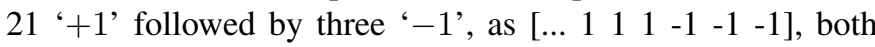
ACF output $\mathbf{B}$ and $\mathbf{D}$ will have output as $\left[\begin{array}{lllllll}\ldots & 1 & 1 & 1 & -1 & -1 & -1\end{array}\right]$.

\section{PERFORMANCE OF THE ACF-BASED SYNCHRONIZER}

\section{A. Performance of Signal Detection and TFC Identification}

We compare the performance of the proposed ACF-based detector with the CCF-based one. Figure 4 shows the miss and false-alarm probabilities for both schemes under different thresholds, on UWB CM1 channels with zero CFO [5], where the values of the threshold are relative to the (average) peak power of the received signal when the preamble signal presents. In SNR ranges of practical interest $(\geq-6 \mathrm{~dB})$, the ACF-based parallel signal detector has a comparable performance to the CCF-based one. (Comparisons are done with optimal threshold levels set for both designs, i.e., 0.2 relative to the peak value for ACF-based detector and 0.7 for $\mathrm{CCF}$ based detector). In addition to the higher computation cost, the performance of CCF-based detector is more sensitive to CFO effect that the ACF-based detector [13]. This makes our the ACF-based parallel signal detector more attractive.

The performance of the proposed parallel signal detector can be quantified. For the (absolute) detection threshold of $\lambda(>0)$, we consider the ACF on two received signal segments with the length $N+N_{g}=160$ and a separation of $p N_{s} T, p=$ $1,3,5,6$ (see Fig. 1). For the output ACF value which is close to $\max _{d}\left\{\left|A C\left(d ; p, N_{g}\right)\right|\right\}$, we find that [16]

1) when synchronization symbols are present in both signal segments. The detection probability is given by

$$
P_{d} \approx Q\left(\frac{\sqrt{2\left(N+N_{g}\right)} \kappa S N R_{r}}{\sqrt{2 \kappa S N R_{r}+1}}, \frac{\sqrt{2 /\left(N+N_{g}\right)} \lambda}{\sigma_{v}^{2} \sqrt{2 \kappa S N R_{r}+1}}\right)
$$

where $S N R_{r}$ is the received SNR, $\kappa=3$ for TFC 1-4 and $\kappa=1$ for TFC $5-7$; and $Q(\cdot, \cdot)$ is the generalized Marcum's Q function [8];

2) when the synchronization symbol is presents in only one of the two signal segments. The false alarm probability can be approximated as

$$
P_{f, 01} \approx \exp \left[-\frac{1}{\left(N+N_{g}\right)} \frac{\lambda^{2}}{\sigma_{v}^{4}\left(\kappa S N R_{r}+1\right)}\right]
$$

3) when no synchronization symbol is present in either signal segment. The false alarm probability is

$$
P_{f, 00}=\exp \left[-\frac{\lambda^{2}}{\left(N+N_{g}\right) \sigma_{v}^{4}}\right]
$$

In the proposed parallel signal detector, we can estimate the probability to detect the signal and correctly identify the TFC group, when the synchronization symbols are present. Since we have 24 repeated synchronization symbols in a preamble [1], this probability can be approximated by

$$
\mathcal{P}_{d}=1-\left[1-\left(1-P_{f, 01}\right)^{2} P_{d}^{2}\right]^{6}
$$

for TF code $1-4$ where 8 synchronization symbols are on each frequency sub-band; and

$$
\mathcal{P}_{d}=1-\left[1-4\left(1-P_{d}\right) P_{d}^{3}-P_{d}^{4}\right]^{18}
$$

for TF code $5-7$ where all 24 synchronization symbols are in the same band. Here the criterion for determining the presence of the synchronization symbol sequence with TFC 5-7 is that at least three out of four outputs (i.e., $[A, B, C, D]$ ) of the parallel signal detector exceed the given threshold. Figure 5 shows that the analytical missed detection probability (i.e., $1-\mathcal{P}_{d}$ ) matches the simulation results very well.

Similarly we can characterize the performance of ACFbased TFC identification with band-switching in TFC 1-4. Consider the ACF output on two received signal segments with the length $N+N_{g}=160$. For the case when synchronization symbols are present in both signal segments, the distribution of the peak (absolute) ACF value $X_{1}$ is given by [16]

$$
f_{X_{1}}(x)=\frac{x}{\sigma_{\nu_{d}}^{2} / 2} \exp \left[-\frac{x^{2}+A_{d}^{2}}{\sigma_{\nu_{d}}^{2}}\right] I_{0}\left(\frac{A_{d} x}{\sigma_{\nu_{d}}^{2} / 2}\right)
$$

where $A_{d} \triangleq \sum_{m}\left|\sum_{i} h_{i} s[m+d-i]\right|^{2}, \sigma_{\nu_{d}}^{2}=\left[2 A_{d}+(N+\right.$ $\left.\left.N_{g}\right) \sigma_{\nu}^{2}\right] \sigma_{\nu}^{2}$ and $I_{0}(\cdot)$ is the zero-order modified Bessel function of the first kind. For the case when no synchronization symbol is present in either signal segment of the ACF, the distribution of the peak (absolute) ACF value $X_{2}$ is [16]

$$
f_{X_{2}}(x)=\frac{x}{\left(N+N_{g}\right) \sigma_{\nu}^{4} / 2} \exp \left\{-\frac{x^{2}}{\left(N+N_{g}\right) \sigma_{\nu}^{4}}\right\} .
$$

Therefore, the error probability in identifying the TFC is

$$
\begin{aligned}
P\left[X_{1}<X_{2}\right] & =\int_{0}^{\infty} P\left[X_{2}>x \mid x\right] f_{X_{1}}(x) d x \\
& \approx \frac{1}{2\left(\kappa S N R_{r}+1\right)} \exp \left[-\frac{\left(N+N_{g}\right) \kappa^{2} S N R_{r}^{2}}{2\left(\kappa S N R_{r}+1\right)}\right](11)
\end{aligned}
$$

where $S N R_{r} \approx \frac{A_{d}}{\kappa\left(N+N_{g}\right) \sigma_{\nu}^{2}}$. When $S N R_{r}<<1$, the decrease of the error probability is approximately proportional to $\exp \left[-c_{1} S N R_{r}^{2}\right]$ where the constant $c_{1} \triangleq\left(N+N_{g}\right) \kappa^{2} / 2$; when $S N R_{r}>>1$, the decrease of the error probability is approximately proportional to $S N R_{r}^{-1} \exp \left[-c_{2} S N R_{r}\right]$ where the constant $c_{2} \triangleq c_{1} / \kappa$. The effectiveness of (11) is verified in Fig. 6. 

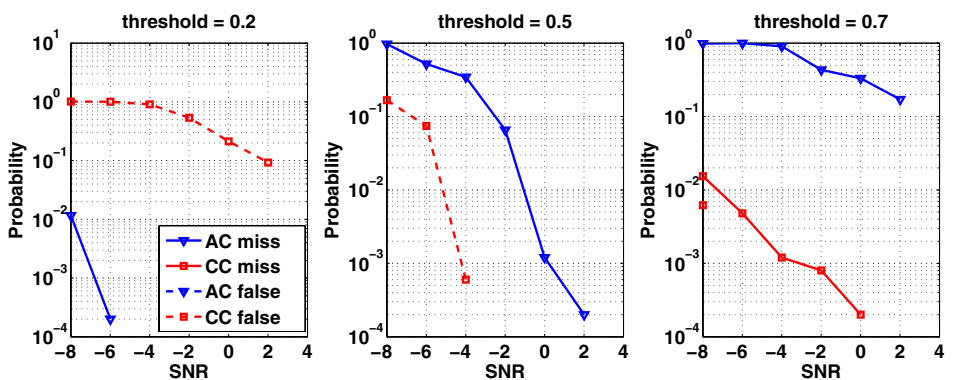

Fig. 4. Miss/False detection probabilities of the parallel ACF-based signal detector (auto-correlation between received signal segments with a length $N+N_{g}=160$ ) and a CCF-based signal detector (CC), in UWB CM1 channel [5] and the TFC number of the received signal is 1. The false-alarm probabilities of the parallel ACF-based signal detector are too low to be shown $\left(<10^{-4}\right)$ in the interested SNR range.
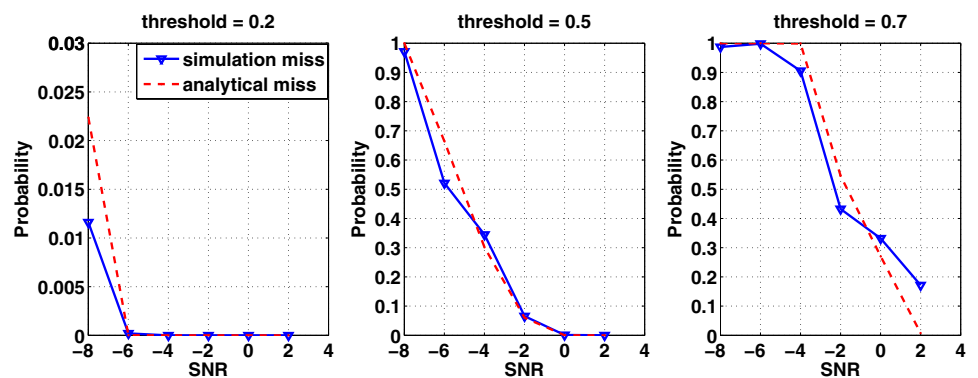

Fig. 5. The comparison of analytical miss detection probabilities $1-\mathcal{P}_{d}$ and the simulation results, the TFC number of the received signal is 1 and the UWB channel is CM1.

\section{B. Performance of OFDM Symbol Timing}

In Fig. 7, we show that the BER performance of the receiver using timing metric (8) is very close to the one with the optimal timing in (5), in both CM1 and CM4 channels. In our design, the timing metric (8) is calculated using the ACF outputs that are already available in signal detection and TFC identification stage and therefore it minimizes the extra resources needed for timing computation.

\section{Performance of Iterative CFO Estimation}

In Fig. 8, we show the residual CFO after the first three iterations of the proposed iterative $\mathrm{CFO}$ estimator under different channels and TFC settings. We see that two iterations are sufficient to reduce the residual $\mathrm{CFO}$ to $1 \sim 2 \mathrm{ppm}$ even when SNR is as low as $-3 d B$. As these ACF outputs are available directly from the parallel ACF block, the proposed $\mathrm{CFO}$ estimation scheme incurs minimum computation burden.

The analysis on the performance of the proposed iterative CFO estimation scheme shows that the error on the current estimation $\Delta \hat{f}+\delta \hat{f}$ can be approximated as [16]

$E[|(\Delta \hat{f}+\delta \hat{f})-\Delta f|] \approx \frac{\sqrt{\left(2+S N R_{r}^{-1} / \kappa\right) S N R_{r}^{-1} / \kappa}}{2 \pi^{3 / 2} p N_{s} \sqrt{N+N_{g}} T}$.

From (12), we can see that the (expected) residual CFO decreases with $S N R_{r}^{-1 / 2}$ when $S N R_{r}>>\frac{1}{2 \kappa}$ (e.g., $S N R_{r}>>$ $-7.8 d B$ for the case TFC $1-4$ where $\kappa=3$ ). The simulation in Fig. 9 confirms the effectiveness of the approximation (12) where the slopes of the residual CFOs decrease in different iterations of the simulation match the prediction by analysis when $S N R_{r} \geq 0 \mathrm{~dB}$. Eqn. (12) also implies that the

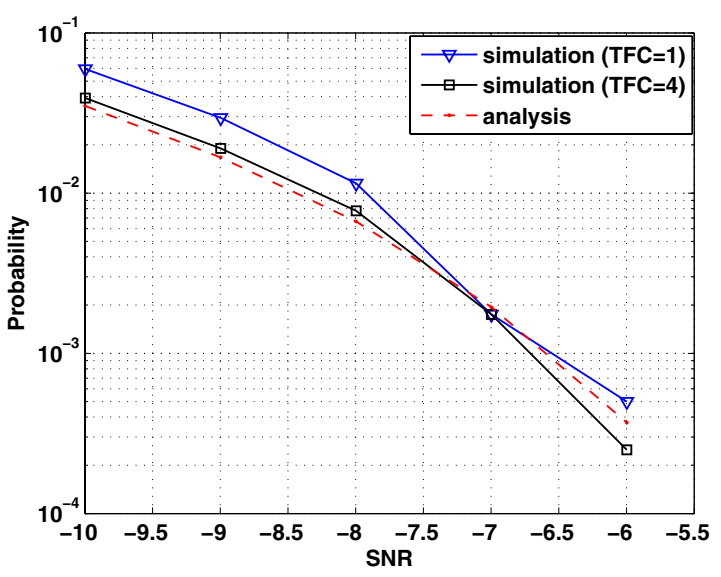

Fig. 6. The TFC identification error probabilities of using band-switching, given that the correct TFC group has been identified in signal detection phase; the analytical result (11) is compared to the simulation result; the UWB channel is CM1.

decrease of residual CFO is proportional to $1 / p$. Therefore, the algorithm (in Table III) requires that $p_{k}>p_{k-1}$ to ensure a decrease of the expected residual $\mathrm{CFO}$ in each iteration.

\section{CONCLuSions}

We have presented a complete synchronization design for MB-OFDM UWB systems featuring a parallel ACF-based structure and all functional units designed using the ACF outputs. The key features of our design include: (1) a joint signal detection and TFC identification with the proposed parallel ACF-based architecture that has a very low computation 


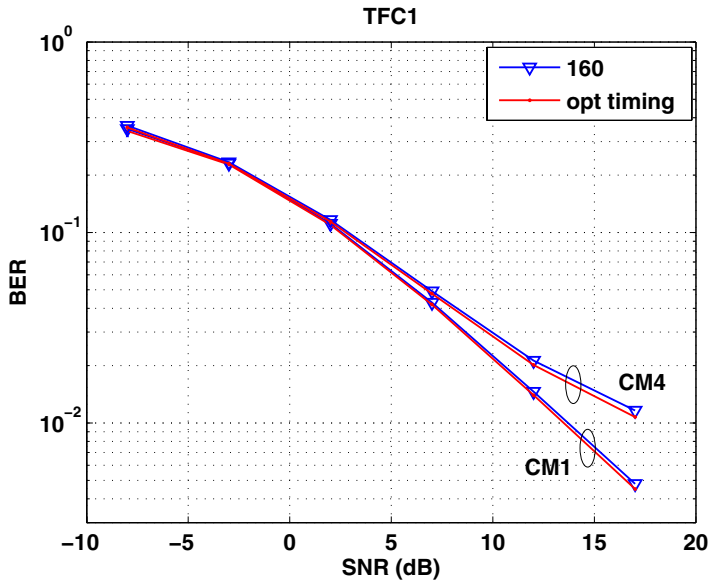

Fig. 7. The demodulated BER performance of using symbol timing metric (8), i.e., " 160 ", compared to the one using the optimal timing point which maximizes the SNR at the output of the FFT operation.
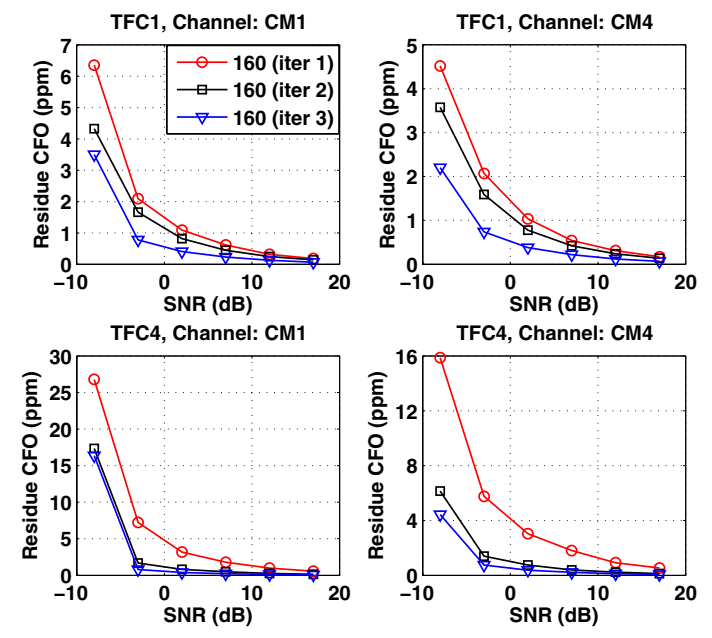

Fig. 8. The residual CFO (in ppm) during the first three iterations of the iterative CFO estimation, under different channel types (CM1, CM4) and different TFCs ( 1 and 4$) ; p=3,6,12$ in three iterations in TFC-1 and $p=1,6,12$ in three iterations in TFC-4; initial CFO is set as $40 \mathrm{ppm}$ at $f_{c}=3.960 \mathrm{GHz} ;$; "160" stands for the length of signal segments in ACF operations $\left(N+N_{g}=160\right)$; the stepsize $\alpha=1$.

cost, fast acquisition and non-compromised performance; (2) a symbol timing with the maximum correlation (MC) metric that can achieve a near-optimal performance, confirmed by our analysis and simulation; (3) an iterative structure for CFO estimation and correction that covers the largest CFO estimation range and achieves the highest accuracy simultaneously. Most importantly, all the designs described in this paper can be readily and efficiently implemented in hardware.

\section{ACKNOWLEDGMENT}

This work is funded by Renesas Technology Corp.

\section{REFERENCES}

[1] ECMA, "High Rate Ultra-Wideband PHY and MAC Standard", Standard ECMA-368, 1st Ed., Dec. 2005.

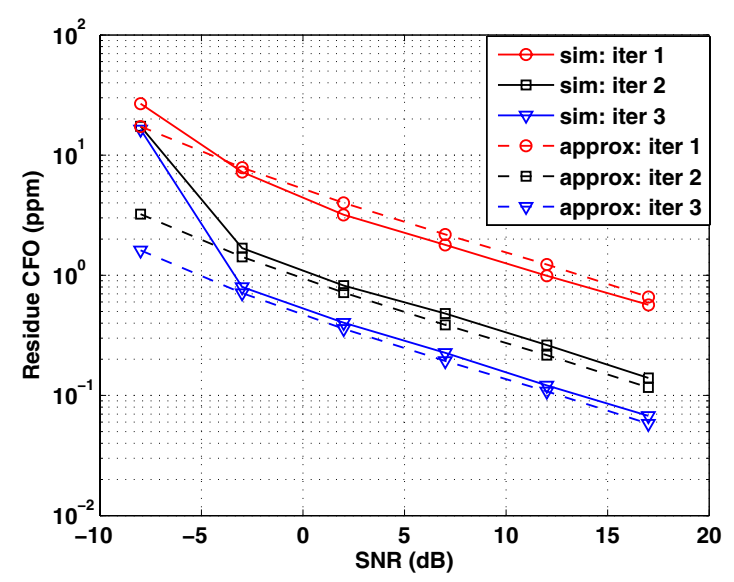

Fig. 9. The residual CFO (in ppm) in the first three iterations in the iterative CFO estimation and correction, compared to the approximation in (12); the TFC number is 4 and the channel is CM1; the stepsize $\alpha=1$.

[2] M. Speth, S. A. Fechtel, G. Fock, H. Meyr, "Optimum Receiver Design for Wireless Broad-Band Systems Using OFDM - Part I", IEEE Trans. on Commun., Vol. 47, No. 11, pp.1668-1677, Nov. 1999.

[3] M. Speth, S. A. Fechtel, G. Fock, H. Meyr, "Optimum Receiver Design for OFDM-Based Broadband Transmission - Part II: A Case Study", IEEE Trans. on Commun., Vol. 49, No. 4, pp.571-578, Apr. 2001.

[4] A. J. Coulson, "Narrowband interference in pilot symbol assisted OFDM systems", IEEE Trans. Wireless Commun., vol. 3, no. 6, pp. 2277-2287, Nov. 2004.

[5] A. F. Molisch, J. R. Foerster, and M. Pendergrass, "Channel models for ultrawideband Personal Area Networks", IEEE Personal Communications Magazine, no. 55, pp. 14-21, 2003.

[6] T. Schmidl and D. Cox, "Robust frequency and timing synchronization for OFDM", IEEE Trans. Commun, vol. 45, no.12, 1613-1621, 1997.

[7] H. Minn, V. Bhargava, and K. Lataief, "A robust timing and frequency synchronization for OFDM systems," IEEE Trans. Wireless Commun., vol. 2, no. 4, pp. 822-838, Jul. 2003.

[8] J. G. Proakis, Digital Communications, 3rd ed. McGraw-Hill, Inc., 1995, pp. 41-48.

[9] S. H. Muller-Weinfurtner, "On the Optimality of Metrics for Coarse Frame Synchronization in OFDM: a Comparison", IEEE PIMRC '98, 1998.

[10] K. Shi, Y. Zhou, B. Kelleci, T. W. Fischer, E. Serpedin, A. lker Karsilayan, "Impacts of Narrowband Interference on OFDM-UWB Receivers: Analysis and Mitigation", IEEE Trans. on Signal Processing, Vol. 55, no. 3, pp. 1118-1128, Mar. 2007.

[11] C.R.N. Athaudage; R.R.V. Angiras, "Sensitivity of FFT-equalised zeropadded OFDM systems to time and frequency synchronisation errors", IEE Proc. on Commun., Vol. 152, no. 6, pp. 945-951, Dec. 2005.

[12] H. Liu and C. Lee, "A Low-Complexity Synchronizer for OFDM-Based UWB System", IEEE Trasn. On Circuits and Systems-Part II, Vol. 53, no. 11 , pp. $1269-1273$, Nov. 2006

[13] M. Krstic, A. Troya, K. Maharatna and E. Grass, "Optimized low-power synchronizer design for the IEEE 802.11(a) standard", IEEE ICASSP 2003, pp. 333-336, Hong Kong, 2003.

[14] F. Tufvesson, O. Edfors, and M. Faulkner, "Time and frequency synchronization for OFDM using PN-sequence preambles," in IEEE VTC, vol. 4, pp. 2203-2207, Amsterdam, The Netherlands, Sept. 1999.

[15] S. YOON and J. CHONG, "Packet Detection and Symbol Timing Synchronization Algorithm for Multi-Band OFDM UWB", IEICE Trans. on Commun., Vol.E89-B No.4 pp.1433-1435, Apr. 2006.

[16] Z. Ye, C. Duan, P. Orlik and J. Zhang, "A Low-Complexity Synchronizer Design for MB-OFDM Ultra-wideband (UWB) Systems", Technique Report, Mitsubishi Electric Research Laboratories, Aug. 2007. 